\title{
sciendo
}

\section{Chain claims reserving methods in non-life insurance}

\author{
Aurora Elena DINA (MANOLACHE) \\ University of Economic Studies, Bucharest, Romania \\ Auroradina86@yahoo.com
}

\begin{abstract}
Considering that the reliability of reserves valuation directly influences the financial strength of an insurance company, the main aim of this paper is to present a claims reserving estimation for a Romanian non-life insurer based on the most popular chain methods which are typically used in practice for the estimation of outstanding claims reserves in general insurance industry: Standard Chain Ladder and Munich Chain Ladder both on the claims incurred data and claims paid data. The tail development factors have been estimated based on the curve-fitting methods. The obvious advantage of these methods is represented by its simplicity of the practicality application. The results of the research under two chain claims reserving models reveal significant differences between the Standard Chain Ladder and Munich Chain Ladder with respect to the claims reserves level. Probably the Standard Chain Ladder based on paid method underestimates the outstanding loss liabilities and Standard Chain Ladder based on Incurred method overestimates the claims reserves. The claims reserves predictions under the Paid Munich Chain Ladder and Incurred Munich Chain Ladder are between the two Standard Chain Ladder outstanding loss liabilities estimates. The results of the tail extrapolation shown that the incorporation of the tail factors can have a significant impact on claims predictions.
\end{abstract}

Keywords: Chain-Ladder claim reserving, non-life insurance run-off, Munich Chain Ladder, conditional mean square error, tail factor.

\section{Introduction}

The job of a reserving actuary is to predict, based on historical data analysis and expert judgement with maximum accuracy, the total amount necessary to pay for the claims generated by the contracts sold to clients. There are two types of reserves: premium reserves for future exposures and claims reserves for unsettled claims of the past exposures. The claims reserves are split in RBNS (case reserves or outstanding claims, which are losses already reported to the insurance company) and IBNR (losses that are incurred but not reported yet).

Historically, the reserving prediction was based on deterministic calculations with pen and paper, combined with expert judgement. Since the 1980's, when the computer became more powerful, sophisticated models started to be developed. Changes in regulatory requirements, for example the implementation of Solvency II regulatory regime starting with January 1st,2016 by all the European (re)insurers, have promoted to use stochastic and statistical methods to forecast the reserves for insurance business as accurately as possible considering the consequences for the solvency capital evaluation. Over the years, numerous methods and models have been developed to estimate both the level and variability of reserves for insurance company. According to Carrato et al. (2018), in practice the Mack Chain-Ladder and Bootstrap Chain-Ladder models are the most used methods to estimate the reserves across the UK industry and the Lloyd's market.

Reserves (known also as technical provisions) represent the core part of an insurer's 
balance sheet liability and the reliability of their valuation directly influences the financial strength of an insurance company. The annual report published by the Romanian Financial Supervisory Authority for 2017 year shown that the overall reserves for Romanian insurance industry were around 1.88 billion EUR, for which the cost related to the claims reserves is approximatively 1.1 billion EUR, accounting for $59 \%$ of the total. Compared with the previous year, in 2017 the total reserves has recorded an increase by 8\%, of which the claims reserves was grown by $14 \%$.

The main aim of this paper is to present a claims reserving estimation for a Romanian non-life insurer based on the most popular chain methods which are typically used in practice for the estimation of outstanding claims reserves in general insurance industry: Standard Chain Ladder and Munich Chain Ladder both on the claims incurred data and claims paid data. Given the particularly of the Romanian insurance market (non -life dominated accounting for $79 \%$ of total gross written premiums), which is not expected to change significantly over the near term, the results of the papers are relevant for insurance practitioners and academic researchers from the actuarial area.

The article is set out as follows: a brief overview of the literature review is provided in the second section of the paper, the research methodology is shown in the third section of the article, the fourth section contains the results of the claims reserves estimation based on the two methods presented in the methodology section, in the fifth section are outlined the main conclusions with respect to the predictions of the level and variability of claims reserves portfolio analyzed in an appropriate way and in the sixth section it is summarized the bibliography.

\section{Literature review}

The chain ladder method is one of the most popular and well-known methods of claims estimating in non-life insurance. It was developed at a time when computers were not readily available and it was important to have simple closed form expressions. In the past, the classical actuarial literature has described the chain-ladder method as a pure computational algorithm to estimate claims reserves. Since the early 1990s several papers have been published to embed the simple chain ladder method into a statistical framework and started to think about stochastic models which generate the chain-ladder algorithm. Therefore, many extensions of the Classical Chain claims reserving model have started to be developed. Authors such as: Mack $(1993,1994)$ or Murphy $(1994)$ have shown that link ratio techniques for loss reserving can be regarded as weighted regressions of a certain kind. The first who came up with a full stochastic model for the chain-ladder method was Mack. In 1993, Mack published one of the most famous articles in claims reserving on the calculation of the standard error in the chain ladder model. Another key milestone in the development of the chain claims reserving methods was the Munich Chain Ladder (MCL) model developed by Quarg and Mack in 2004. Their aim was to unify claims forecast based on claims payments and incurred losses and reduce the gap between the two chain ladder predictions. Also, Verdier and Klinger (2005), Merz and Wüthrich (2006) and Liu and Verrall (2008) have studied similar stochastic models for claims reserving that allows to combine claims payments and incurred losses information. Merz and Wüthrich (2008) have proposed a multivariate claims reserving method to calculate the age-to-age development factors and to determine the outstanding claims reserve for the different run-off triangles as a whole. 
Verdonck et al. (2009) and Verdonck and Debruyne (2011) have developed stochastic robust version of the chain-ladder method. The papers of Verrall et al. (2010) and Martinez-Miranda et al. (2011) have provided a new chain ladder method for outstanding claims in which was combined the observed incurred count data with the observed paid data known as Double Chain Ladder. In non-life insurance, the claims settlement process goes beyond the latest development period available in the observed claims development triangle and the actuaries have to estimate an additional claim reserve for late payments, so-called tail development factors which account for the unobserved part of the insurance claims. Some of the most popular models applied in practice to estimate the development of tail factors was provided by Mack (1999), Boor (2006) and Verrall and Wüthrich (2012).

\section{Methodology}

In this section are presented the methodologies applied to estimate the claims reserves for a Romanian non-life insurance company. There are tested two methods: Mack Chain Ladder and Munich Chain Ladder under two sets of data: cumulative payments and incurred losses triangles in the claims reserving estimation for insurer analyzed. To determine the tail development factors in the paper were used the curve-fitting methods.

Mack Chain Ladder and Munich Chain Ladder are the most popular chain methods applied by actuarial practitioners in general claims reserving prediction. The obvious advantage of these methods is represented by its simplicity of the practicality application. These reserving methods can be easily implemented in MS Excel, but it would be preferable to be used $\mathrm{R}$ programme which provides a richer language for statistical modelling and data manipulations than MS Excel. R provides also many interfaces to databases and other applications. For example, RExcel is an add-in for Microsoft Excel, which allows access to the statistics package R from within Excel.

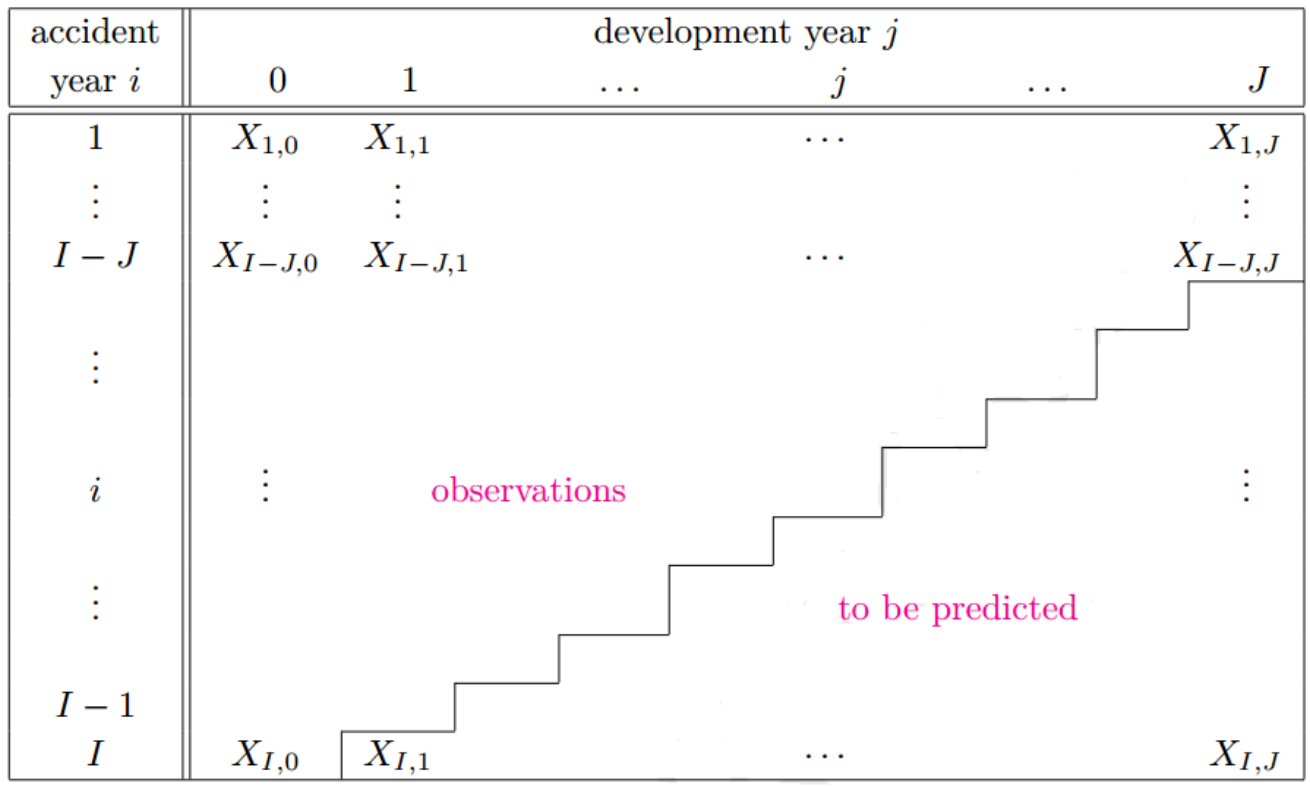

Figure 1. Incremental payments triangle

Source: adopted from Wüthrich and Merz (2015), p.15.

In the chain claims reserving methods, the data sets of the outstanding loss liabilities 
are aggregated into so-called run-off triangles. The claims developments of the run-off triangle contain two time axes: the vertical axis is represented usually by the accident periods and on the horizontal axis are development periods. Run-off triangle is split into two parts: in the upper part of the triangle there are the observations and in the lower part of the triangle there are the outstanding payments which have been predicted. Usually, the upper part of the triangle contains the claims payments or the incurred losses. Incurred losses represent the sum between the cumulative claim payments and case estimates. Figure 1 presents a claims run-off triangle for incremental payments.

Usually, in the claims run-off triangles are used the following notation for accident years $i \in\{0, \ldots ., I)$ and for development years $i \in\{0, \ldots ., J)$ and for simplicity, it was assumed $\mathrm{I}=\mathrm{J}$. In Figure $1, \mathrm{X}_{\mathrm{i}, \mathrm{j}}$ represents the cumulative amount payments of accident year $\mathrm{i}$ and development year $\mathrm{j}$. With $\mathrm{C}_{\mathrm{i}, \mathrm{j}}$ and $\mathrm{I}_{\mathrm{i}, \mathrm{j}}$ (for $1 \leq \mathrm{i}, \mathrm{j} \leq \mathrm{n}$ ) are noted the cumulative amount payments of accident year $i$ and development year $j$, respectively incurred amount payments used to make predictions about the claims that need to be paid in future calendar years. The lower part of the triangle represents the amount that needs to be predicted (the overall reserve that will be paid in the future). The sum between claims reserves and past payments corresponds to the ultimate claim amount for accident year $i\left(C_{i, \infty}\right)$. The outstanding reserve for accident year $\mathrm{i}$ at time $\mathrm{j}\left(\mathrm{R}_{\mathrm{i}, \mathrm{j}}\right)$ is determined using the Equation 1:

$$
R_{i, j}=\sum_{k=j+1}^{\infty} X_{i, k}=C_{i, \infty}-C_{i, j}
$$

Chain-ladder algorithm. The most used model to predict the future claims reserve is the chain-ladder method, which forecasts the cumulative claims recursively applying a set of chain ladder factors (known also: age-to-age factors or link ratios): $\{f \mid j=2, \ldots, n\}$. It assumes that the proportional developments of claims from one development period to the next are the same for all origin years. The chain ladder factors are estimated by applying the Equation 2:

$$
f_{j}=\frac{\sum_{i=1}^{n-j+1} C_{i, j}}{\sum_{i=1}^{n-j+1} C_{i, j-1}}, \text { for } \quad 2 \leq j \leq n
$$

The chain ladder factors are then applied to the latest known cumulative claims amount to forecast the next development period based on the Equations 3 and 4:

$$
\begin{gathered}
C_{i, n-i+2}=C_{i, n-i+1} f_{n-i+2}, \text { for } 1 \leq j \leq n \\
C_{i, k}=C_{i, k-1} f_{k}, \text { for } 1 \leq j \leq n, \quad n-i+3 \leq k \leq n
\end{gathered}
$$

The first method used in the research claims reserving forecast is the distribution-free chain ladder model of the standard error in the chain ladder model developed by Mack in 1993. The method forecasts the standard errors of the chain-ladder estimation without assuming a distribution under three conditions: (1) expectation assumption, (2) variance assumption and (3) independence assumption. If these assumptions are hold, the Mack chain-ladder model gives an unbiased estimator for claims reserves. The detailed methodology of the Mack's distribution-free CL model and conditional mean square error of prediction (MSEP) is presented in the paper of Mack (1993).

The second method used in the paper is the Munich Chain Ladder (MCL) model based on claims payments and incurred losses developed by Quarg and Mack in 2004 with the goal to reduce the gap between the two chain ladder predictions which are based on claims 
payments and incurred losses data, respectively. The detailed methodology for prediction of the outstanding loss liabilities under Munich Chain Ladder method is defined in the paper of Quarg and Mack (2004).

To estimate the tail development factors in a mathematically consistent way, in the research were used the curve-fitting methods. These models for developing tail factors consist into positioning some relationship between the link ratios at various development ages and use that relationship as an assumption to fit a curve to the link ratios, with the aim to generate the projected link ratios in the development ages covered by the tail factor. All those projected link ratios can then be multiplied together to provide an estimate of the tail factor. In order to extrapolate the tail development factors, there have been used four distributions which are usually applied in practice for modeling a decay process: Exponential, Sherman, Weibull and Power distributions. The detailed methodology for tail factors estimation using curve-fitting methods is shown in the paper of Boor (2006).

\section{Results and discussions}

In the Table 1 and Table 2 there are the two data sets of the paid and incurred run-off triangles used in the claims reserves estimation of the portfolio for the Romanian non-life insurance company analyzed. The triangles cover seven accident years and thus seven developments years. In the last row of each table there are the chain ladder factors.

Table 1: Cumulated payments triangle (EUR thousand)

\begin{tabular}{|l|r|r|r|r|r|r|c|}
\hline $\begin{array}{c}\text { Development } \\
\text { years / } \\
\text { Accident Years }\end{array}$ & $\mathbf{1}$ & $\mathbf{2}$ & $\mathbf{3}$ & $\mathbf{4}$ & $\mathbf{5}$ & $\mathbf{6}$ & $\mathbf{7}$ \\
\hline 2011 & 117.395 & 175.996 & 182.244 & 186.068 & 188.194 & 189.862 & 191.283 \\
\hline 2012 & 125.201 & 167.999 & 175.092 & 178.779 & 181.796 & 184.375 & \\
\hline 2013 & 91.956 & 122.609 & 127.445 & 130.586 & 132.214 & & \\
\hline 2014 & 89.353 & 130.166 & 137.131 & 143.283 & & & \\
\hline 2015 & 76.906 & 116.139 & 121.373 & & & & \\
\hline 2016 & 68.839 & 99.361 & & & & & \\
\hline 2017 & 52.813 & & & & & & \\
\hline CL factors & 1,4259 & 1,0426 & 1,0270 & 1,0137 & \multicolumn{7}{|c|}{1,0115} & \multicolumn{2}{c|}{1,0075} & \\
\hline
\end{tabular}

Table 2: Incurred triangle (EUR thousand)

\begin{tabular}{|l|c|c|c|c|c|c|c|}
\hline $\begin{array}{c}\text { Development } \\
\text { years / } \\
\text { Accident Years }\end{array}$ & $\mathbf{1}$ & $\mathbf{2}$ & $\mathbf{3}$ & $\mathbf{4}$ & $\mathbf{5}$ & $\mathbf{6}$ & $\mathbf{7}$ \\
\hline 2011 & 164.340 & 190.744 & 194.396 & 196.364 & 193.614 & 193.774 & 194.346 \\
\hline 2012 & 170.793 & 186.991 & 188.978 & 185.222 & 187.412 & 187.980 & \\
\hline 2013 & 127.974 & 142.877 & 138.654 & 138.498 & 139.613 & & \\
\hline 2014 & 140.038 & 152.466 & 150.187 & 153.796 & & & \\
\hline 2015 & 116.749 & 128.472 & 130.594 & & & & \\
\hline 2016 & 102.663 & 117.257 & & & & & \\
\hline 2017 & 86.776 & & & & & & \\
\hline CL factors & 1,1170 & 1,0016 & 1,0025 & 1,0011 & 1,0019 & 1,0030 & \\
\hline
\end{tabular}

Source: Authors' own research

Before calculation of the claims reserves, there needs to be detected the claims that 
have an abnormally positive or negative influence on the reserve estimation. It is well known that the chain-ladder method is very sensitive to outlying data and even one outlier can lead to a huge over or underestimation of the overall reserve when using the chain-ladder method. This indicates that individual claims can be very influential when determining the chainladder estimation. However, the decision of choosing the outstanding claims reserve with or without outliers remains a problem for the risk management of an insurance company. In the detection of the outliers in the data set, the univariate methods are often favored due to their simplicity but to estimate a realistic reserve it is preferred to use a robust stochastic model by calculating the influence functions in the generalized linear model framework corresponding to the chain-ladder method such as that has been proposed by Verdonck and Debruyne (2009) or the robust versions to replace the classical reserving techniques proposed by Verdonck and Van Wouwe (2011). The individual factors (IF) can also be used as a diagnostic tool to visualize the influential observations. Figure 2 presents the evolution of the individual development factors of paid and incurred run-off triangles:

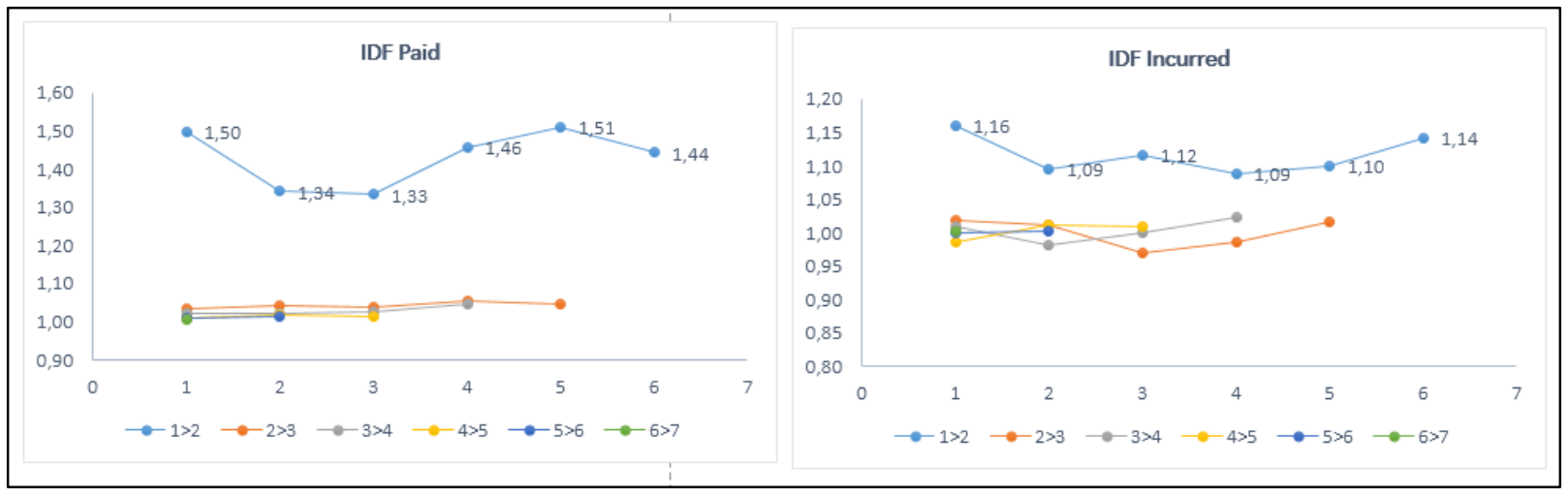

Figure 2. Individual development factors (IDF)

Source: Authors' own research.

Table 3 shows the average, minimum, maximum, standard deviation and coefficient of variation of the individual development factors:

Table 3: Individual development factors (IDF)

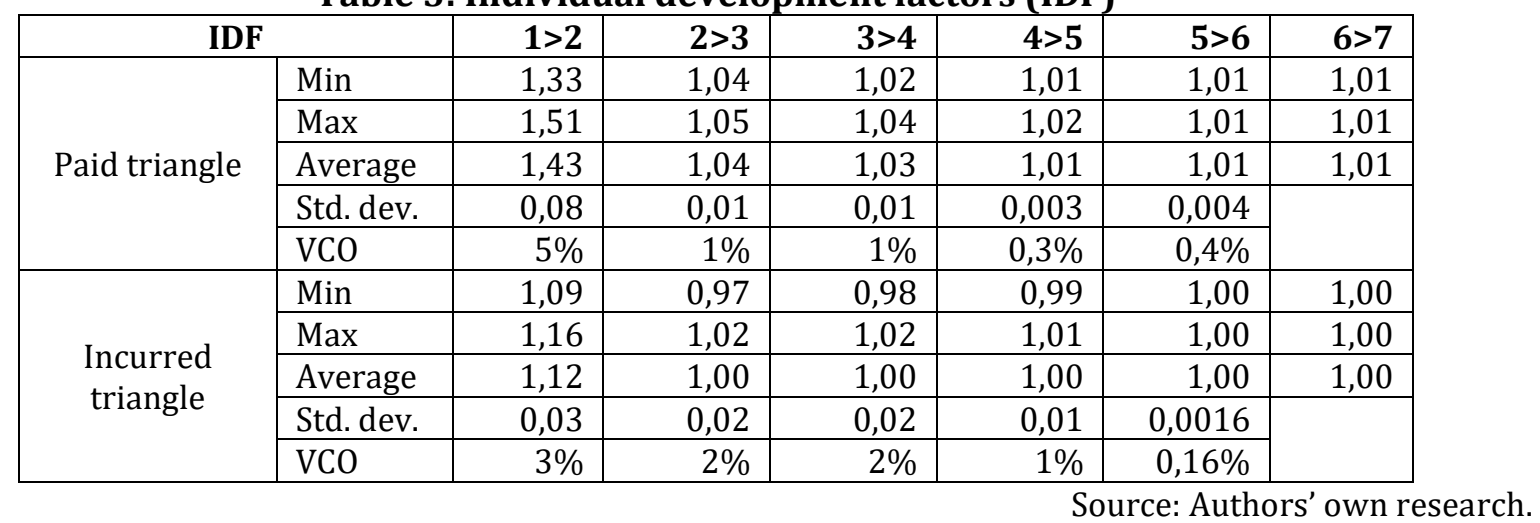

After a closer look at the graphically individual factors development, can be observed an oscillation of the factors (some points are different from the majority of the data). These outliers are not incorrect (are not due to the copying or recording of mistakes), they are big 
claims which were made under exceptional circumstances for motor third part liability and fire lines of business. Even if the outliers do not fit with the model well, prudentially the run - off triangles have been used without any further adjustment. The decision to keep the atypical value of this observations was based on the reason that such an extreme claims events will happen again.

In the Table 4, there are presented the results of the claims reserves predictions based on the: Standard Chain-Ladder (SCL) and Munich Chain Ladder (MCL) methods both on the claims paid data and claims incurred data.

Table 4: Claims reserves estimation under SCL and MCL (EUR thousand)

\begin{tabular}{|r|r|r|r|r|}
\hline $\begin{array}{c}\text { Accidenty } \\
\text { ear }\end{array}$ & \multicolumn{1}{c|}{$\begin{array}{c}\text { Reserves } \\
\text { SCLPaid }\end{array}$} & \multicolumn{1}{c|}{$\begin{array}{c}\text { Reserves } \\
\text { SCLIncurred }\end{array}$} & \multicolumn{1}{c|}{$\begin{array}{l}\text { ReservesM } \\
\text { CLPaid }\end{array}$} & \multicolumn{1}{c|}{$\begin{array}{c}\text { Reserves } \\
\text { MCLIncurred }\end{array}$} \\
\hline 1 & 0 & 3.064 & 0 & 3.064 \\
\hline 2 & 1.379 & 4.161 & 1.214 & 4.169 \\
\hline 3 & 2.518 & 8.080 & 7.963 & 7.722 \\
\hline 4 & 4.724 & 11.427 & 10.115 & 10.197 \\
\hline 5 & 7.390 & 10.322 & 9.597 & 10.514 \\
\hline 6 & 10.541 & 19.070 & 14.515 & 15.498 \\
\hline 7 & 30.483 & 45.088 & 38.382 & 39.236 \\
\hline Total & 57.035 & 101.212 & 81.786 & 90.399 \\
\hline
\end{tabular}

Source: Authors' own research.

he classical chain-ladder method based on the paid data gives an overall claims reserves estimation of 57 million EUR. The projection based on the incurred is far different than the projection based on the paid losses. The overall reserve estimation of incurred losses have generated a higher reserve projection than paid losses with 44 million EUR.

Table 5 shows the conditional MSEP for the ultimate claim estimates from the distribution-free Chain Ladder model of Mack: the estimated conditional process standard deviation, parameter error ${ }^{1 / 2}$ and the conditional MSEP for the each accident year and aggregate accident years. The aim of the Mack's MSEP is to provide a run-off pattern of the total prediction uncertainty. For the total claims reserves the rooted conditional MSEP is about $14 \%$ of the total claims reserves under Paid Chain Ladder method, respectively 10\% under Incurred Chain Ladder method.

Table 5: MSEP using Mack's formula under SCL based on paid and incurred (EUR thousand)

\begin{tabular}{|c|c|c|c|c|c|c|c|c|c|c|}
\hline \multirow{2}{*}{$\begin{array}{l}\text { Acci } \\
\text { dent } \\
\text { year }\end{array}$} & \multicolumn{5}{|c|}{ SCLpaid } & \multicolumn{5}{|c|}{ SCLincurred } \\
\hline & $\begin{array}{c}\text { Reserve } \\
\text { s }\end{array}$ & $\begin{array}{c}\text { proces } \\
\text { sstd.d } \\
\text { ev. }\end{array}$ & $\begin{array}{l}\text { param } \\
\text { eterer } \\
\text { ror }^{1 / 2}\end{array}$ & $\begin{array}{c}\text { Mack } \\
\text { MSEP1/2 }\end{array}$ & $\begin{array}{l}\text { \%res } \\
\text { erve } \\
\text { s }\end{array}$ & $\begin{array}{c}\text { Reserve } \\
\text { s }\end{array}$ & $\begin{array}{c}\text { proc } \\
\text { essst } \\
\text { d.de } \\
\text { v. }\end{array}$ & $\begin{array}{c}\text { parame } \\
\text { tererror } \\
1 / 2\end{array}$ & $\begin{array}{l}\text { Mack } \\
\text { MSEP1/ } \\
2\end{array}$ & $\begin{array}{c}\% \text { re } \\
\text { serv } \\
\text { es }\end{array}$ \\
\hline 1 & 0 & 0 & 0 & 0 & $0 \%$ & 3.064 & 0 & 0 & 0 & 0 \\
\hline 2 & 1.379 & 523 & 515 & 734 & $53 \%$ & 4.161 & 33 & 33 & 47 & $1 \%$ \\
\hline 3 & 2.518 & 741 & 515 & 902 & $36 \%$ & 8.080 & 257 & 156 & 301 & $4 \%$ \\
\hline 4 & 4.724 & 908 & 619 & 1.099 & $23 \%$ & 11.427 & 2.393 & 1.304 & 2.725 & $24 \%$ \\
\hline 5 & 7.390 & 1.785 & 879 & 1.990 & $27 \%$ & 10.322 & 3.561 & 1.658 & 3.929 & $38 \%$ \\
\hline 6 & 10.541 & 1.859 & 816 & 2.030 & $19 \%$ & 19.070 & 4.455 & 1.860 & 4.827 & $25 \%$ \\
\hline 7 & 30.483 & 6.388 & 1.981 & 6.688 & $22 \%$ & 45.088 & 5.209 & 1.870 & 5.534 & $12 \%$ \\
\hline Total & 57.035 & 7.007 & 3.853 & 7.997 & $14 \%$ & 101.212 & 8.090 & 5.833 & 9.974 & $10 \%$ \\
\hline
\end{tabular}


The claims reserves predictions under the Paid Munich Chain Ladder and Incurred Munich Chain Ladder are between the two Standard Chain Ladder methods. The gap between paid and incurred reserves estimation under Munich Chain Ladder method is five times smaller than between the two predictions of the Standard Chain Ladder method, approximately 8.6 million EUR. The Munich Chain Ladder method was specially designed to remedy the gap between the two chain ladder predictions by adjusting the Chain Ladder factors with incurred-paid ratios. For the recent accident years the payments are usually low and the most amounts are in the case reserve estimates and the main advantage of the Munich Chain Ladder method is the use of the reserving information included in the incurred amounts to improve for the paid losses.

Usually in non-life insurance, the claims settlement process goes beyond the latest development period available in the observed claims development triangle and it is necessary to estimate so-called tail development factors which account for the unobserved part of the insurance claims. By analyzing the chain ladder factors of both run-off triangles (paid and incurred) presented in the A. Appendix, it can be observed that not all claims are settled and it should be built an additional reserve for the late payments beyond the last observed development year of the run-off triangles. In order to forecast the potential further developments beyond development age 7 , the tail factors have been developed based on the four distributions: Exponential, Sherman, Weibull and Power. Table 6 presents the results of the tail factors extrapolation.

Table 6: Tail extrapolation results

\begin{tabular}{|l|r|r|r|r|}
\hline \multirow{2}{*}{ Distribution } & \multicolumn{2}{|c|}{ Paid Chain Ladder } & \multicolumn{2}{c|}{ Incurred Chain Ladder } \\
\cline { 2 - 5 } & Tail Factor & $\mathbf{R}^{\mathbf{2}}$ & Tail Factor & \multicolumn{1}{c|}{$\mathbf{R}^{\mathbf{2}}$} \\
\hline Exponential & 1,0047 & $51,03 \%$ & 1,0014 & $3,56 \%$ \\
\hline Sherman & 1,0283 & $89,28 \%$ & 1,0049 & $34,01 \%$ \\
\hline Weibull & 1,0043 & $86,00 \%$ & 1,0008 & $55,72 \%$ \\
\hline Power & 1,0052 & $49,42 \%$ & 1,0014 & $2,98 \%$ \\
\hline
\end{tabular}

Based on the coefficient of determination (R-square) criteria, the Sherman distribution for Paid Chain Ladder Factors and Weibull distribution for Incurred Chain Ladder Factors were considered to better fit in the tail factors extrapolation. The incorporation of tail development factors have led to a substantial increase of the claims reserves estimate under the Standard Chain Ladder and Munich chain Ladder methods based on Paid with approximatively 28 million EUR. Table 7 presents the results of the claims reserves predictions of the analyzed portfolio without tail factors and with tail factors.

Table 7: Claims Reserves without and with Tail (EUR thousand)

\begin{tabular}{|l|r|r|r|}
\hline \multicolumn{1}{|c|}{ Methods } & $\begin{array}{c}\text { Reserves } \\
\text { No Tail }\end{array}$ & $\begin{array}{c}\text { Reserves } \\
\text { incl. Tail }\end{array}$ & Differences \\
\hline Standard Chain Ladder based on Paid & 57.035 & 84.775 & 27.739 \\
\hline Standard Chain Ladder based on Incurred & 101.212 & 102.054 & 842 \\
\hline Munich Chain Ladder based on Paid & 81.786 & 110.225 & 28.439 \\
\hline Munich Chain Ladder based on Incurred & 90.399 & 91.233 & 833 \\
\hline
\end{tabular}

Source: Authors' own research 


\section{Conclusion}

The results of the research under two chain claims reserving models reveal significant differences between the Standard Chain Ladder and Munich Chain Ladder with respect to the claims reserves level. Probably the Standard Chain Ladder based on paid method underestimates the outstanding loss liabilities and Standard Chain Ladder based on incurred method overestimates the claims reserves. The claims reserves predictions under the Paid Munich Chain Ladder and Incurred Munich Chain Ladder are between the two Standard Chain Ladder outstanding loss liabilities estimates. The results of the tail extrapolation shown that the incorporation of the tail factors can have a significant impact on claims predictions.

Considering that the reliability of reserves valuation directly influences the financial strength of an insurance company and have a substantial impact in the solvency capital requirements, it is highly recommended to examine the data and decide which reserve is more accurate. Given that in this paper, the two chain ladder methods have been applied on the overall portfolio of the Romanian non-life insurer analyzed, to predict a realistic reserve, the proposal for the future research of the claims reserves estimation is to split the overall portfolio into several run-off triangles with possible correlation (such as: motor vehicle liability, motor-other classes, property, liability and other lines of business) and then calculate the claims reserves based on the multivariate claims reserving methods. Also, the study of the portfolio characteristics and the detection of atypical observations in a run-off triangle are helpful to predict a more accurate claim reserve.

\section{References}

Boor, J. (2006). Estimating Tail Development Factors: What to Do When the Triangle Runs Out. Casualty Actuarial Society Forum, Winter 2006, 345-390

Carrato, A., Concina, F., Gesmann, M., Murphy, D., Wüthrich M., Zhang, W. (2010). Claims reserving with R: ChainLadder-0.2.9 Package Vignette, available at: https://cran.rproject.org/web/packages/ChainLadder/vignettes/ChainLadder.pdf

Financial Supervision Authority (2018). The evolution of the Romanian insurance market in 2017, available at: www.asf.ro

Liu, H., Verrall, R.J. (2008). Bootstrap estimation of the predictive distributions of reserves using paid and incurred claims. In: Conference Paper, 38th Astin Colloquium 2008. Manchester, UK

Mack, T. (1993). Distribution-free calculation of the standard error of chain ladder reserve estimates. Astin Bulletin, 23(2), 213-225

Mack, T. (1994). Measuring the Variability of Chain Ladder Reserve Estimates. Casualty Actuarial Society Forum, 1, 101-182

Mack, T. (1999). The Standard Error of Chain Ladder Reserve Estimates: Recursive Calculation and Inclusion of a Tail Factor. Astin Bulletin, 29, 361-366

Martinez-Miranda, M.D., Nielsen, B., Nielsen, J.P., Verrall, R. (2011). Cash flow simulation for a model of outstanding liabilities based on claim amounts and claim numbers. Astin Bulletin, 41(1), 107-129

Martinez-Miranda, M.D., Nielsen, J., Verrall, R. (2012). Double chain-ladder. Astin Bulletin, 42(1), 59-76.

Merz, M., Wüthrich, M.V. (2006), A credibility approach to the Munich chain-ladder method. Blatter DGVFM, 27, 619-628

Murphy, D.M. (1994). Unbiased loss development factors. Proc. CAS, LXXXI, 154-222.

Quarg, G., Mack, T. (2004). Munich chain ladder. Deutschen Gesellschaft fur Versicherungsund Finanzmathematik, 26(4), 597-630 
Verdier, B., Klinger, A. (2005). JAB Chain: A model-based calculation of paid and incurred loss development factors. Conference paper, presented at the 36th International Astin Colloquium, 4-7 September 2005, ETH Zürich, Switzerland

Verdonck, T., Van Wouwe, M., Dhaene, J. (2009). A robustification of the chain-ladder method. North American Actuarial Journal, 13 (2), 280-298

Verdonck, T., Debruyne, M. (2011). The influence of individual claims on the chain-ladder estimates: Analysis and diagnostic tool. Insurance: Mathematics and Economics, 48, 85-98

Verrall, R., Nielsen, J.P., Jessen, A. (2010). Including Count Data in Claims Reserving. Astin Bulletin, 40(2), 871-887

Verrall, R. J., Wüthrich, M. V. (2012). Reversible Jump Markov Chain Monte Carlo Method for Parameter Reduction in Claims Reserving. North American Actuarial Journal, 16, 240-259

Wüthrich, M.V., Merz, M. (2008). Stochastic Claims Reserving Methods in Insurance. Wiley Finance

Wüthrich, M. V., Merz, M. (2015) Stochastic Claims Reserving Manual: Advances in Dynamic Modeling. Swiss Finance Institute Research Paper No. 15-34. Available at https://ssrn.com/abstract=2649057 or http://dx.doi.org/10.2139/ssrn.2649057 